\begin{tabular}{|c|l|}
\hline Title & Transfer matrix method for a dynamical mesoscopic system \\
\hline Author(s) & Y akubo, K. \\
\hline Citation & $\begin{array}{l}\text { PHY SICAL REVIEW E, 57(3), 3602-3610 } \\
\text { https://doi.org/10.1103/PhysRevE.57.3602 }\end{array}$ \\
\hline Issue Date & 1998-03 \\
\hline Doc URL & http://hdl.handle.net/2115/5651 \\
\hline Rights & Copyright $\odot$ 1998 A merican Physical Society \\
\hline Type & article \\
\hline File Information & PRE57-3.pdf \\
\hline
\end{tabular}

Instructions for use 


\title{
Transfer matrix method for a dynamical mesoscopic system
}

\author{
K. Yakubo \\ Department of Applied Physics, Hokkaido University, Sapporo 060-8628, Japan
}

(Received 23 June 1997)

\begin{abstract}
A transfer matrix method for analyzing quantum transport in harmonically driven systems with spatiotemporal coherence has been developed. In such systems, the energy conservation law is violated, and transport occurs through an infinite number of sideband states. This transfer matrix method enables us to compute transmission and reflection probabilities of such dynamical mesoscopic systems without consuming a large amount of computing time and memory space. We apply this technique to photon-assisted tunneling (PAT) in a dual-gate field-effect transistor, and demonstrate the possibility of observing resonant level splittings due to the dynamical Stark effect in dual-gate PAT devices. [S1063-651X(98)06103-0]
\end{abstract}

PACS number(s): 02.70.-c, 73.23.-b, 73.40.Gk, 73.50.Mx

\section{INTRODUCTION}

Quantum transport through mesoscopic systems is an active research field in condensed matter physics. Recent advances in microfabrication technology have made it possible to realize electron transport in harmonically driven systems showing electron coherence $[1,2]$. In such dynamical mesoscopic systems, new effects, such as delocalization in a photonic field [3], the dynamic localization [4], the absolute negative conductance [5], and dephasing by oscillating potential [6], due to the spatiotemporal coherence of the electronic states are expected. Photon-assisted tunneling (PAT) is a typical example of dynamical mesoscopic transport [716]. Since an electronic state in an electromagnetic field of frequency $\omega$ possesses a finite amplitude in an infinite number of sideband states whose energies are separated by $\hbar \omega$, the tunneling current depends on both the frequency and the intensity of the external ac field. In general, the response of the current on applying an ac field is very quick $\left(\sim 10^{-12}\right.$ $\mathrm{sec})$. It may thus be feasible to develop a high-speed detector of electromagnetic waves or a photoinduced switch. Electron transport through vibrating systems is another example of dynamical mesoscopic transport $[17,18]$. In this case, phonon sidebands play an important role in the transport. The coupling between an electron and an external field in a dynamical mesoscopic system must be distinguished from the usual inelastic scattering leading to incoherence of electronic states. In dynamical mesoscopic transport, the phase memory of the electron still remains after the coupling, but the energy transfer occurs, because the external field is coherent.

In theoretical studies of electron transport through static mesoscopic systems, the transfer matrix method is a powerful tool. This method enables us to numerically calculate transmission probabilities for arbitrarily shaped potential barriers. The conductance of the system can be obtained from the transmission probability by using the Landauer formula $[19,20]$. However, since the conventional transfer matrix method fails in systems for which energy conservation is violated, it is inapplicable in the presence of a timedependent external field. In order to calculate numerically the transmission probability of a dynamical mesoscopic system, one has to solve directly the time-dependent Schrödinger equation with a specific initial condition such as a
Gaussian wave packet [21]. The numerical integration of a parabolic partial differential equation such as the Schrödinger equation usually consumes much computing time. For example, the Crank-Nicolson scheme [22] requires a small time slice $\Delta t$ in order to satisfy the unitarity condition, and $\Delta t$ decreases with decreasing spatial interval $\Delta x$. This means that the computing time often becomes excessively long for complex systems or systems used in actual experiments.

In this paper, we develop a transfer matrix method for dynamical mesoscopic systems with harmonically oscillating potentials in addition to arbitrary static potentials. The present technique can lead to a considerable saving of computer resources compared to a numerical approach to the solution of the time-dependent Schrödinger equation. This method enables us to calculate not only the total transmission and reflection probabilities but also the contributions from each sideband state yielded by the oscillating potential. The paper is organized as follows. In Sec. II, the transfer matrix method is formulated for one-dimensional systems. We present the explicit form of the transfer matrix and transmission probability through each sideband in this section. The method described in Sec. II is extended to two-dimensional systems in Sec. III. Quantum transport in a two-dimensional dynamical mesoscopic system occurs through many channels in addition to sideband states. By using the two-dimensional version of the transfer matrix method, it is possible to calculate the transmission probability through the $\nu$ th channel of the $p$ th sideband. In Sec. IV, using the present numerical method, we predict that resonant level splitting phenomena caused by the dynamical Stark effect can occur in doublebarrier PAT devices. Concluding remarks are given in Sec. V.

\section{ONE-DIMENSIONAL SYSTEMS}

In this section, we consider a one-dimensional mesoscopic system with a static and a harmonically oscillating potentials. The basic idea to construct a transfer matrix is similar to that of Ref. [23]. An electron in the system can be described by the Schrödinger equation,

$$
i \hbar \frac{\partial \psi}{\partial t}=\left[-\frac{\hbar^{2}}{2 m^{*}} \frac{\partial^{2}}{\partial x^{2}}+V_{0}(x)+V_{1}(x) \cos \omega t\right] \psi,
$$


where $V_{0}(x)$ and $V_{1}(x)$ are arbitrary functions of $x$. Hereafter we assume that $V_{0}$ approaches finite values asymptotically at $x \rightarrow \pm \infty$, and the origin of the energy scale is chosen to be $V_{0}(-\infty)=0$. The symbol $m^{*}$ represents the effective mass of the electron. In order to formulate a transfer matrix, we divide the system into $N$ segments with a width $\Delta x$. The $n$th segment is a region between $x^{n-1}$ and $x^{n}$. The width $\Delta x$ is so small that the potentials $V_{0}(x)$ and $V_{1}(x)$ can be regarded as constants in each segment. These constant values for $V_{0}(x)$ and $V_{1}(x)$ in the $n$th segment are given by $V_{0}^{n}$ $=\left[V_{0}\left(x^{n-1}\right)+V_{0}\left(x^{n}\right)\right] / 2$ and $V_{1}^{n}=\left[V_{1}\left(x^{n-1}\right)+V_{1}\left(x^{n}\right)\right] / 2$, respectively. The wave function $\psi^{n}(x, t)$ in the $n$th segment is then governed by

$$
i \hbar \frac{\partial \psi^{n}}{\partial t}=\left[-\frac{\hbar^{2}}{2 m^{*}} \frac{\partial^{2}}{\partial x^{2}}+V_{0}^{n}+V_{1}^{n} \cos \omega t\right] \psi^{n} .
$$

The general solution of Eq. (2.2) is given by

$$
\begin{aligned}
\psi^{n}(x, t)= & \int d E\left[A(E) e^{i k^{n}(E) x}\right. \\
& \left.+B(E) e^{-i k^{n}(E) x}\right] \sum_{m=-\infty}^{\infty} J_{m}\left(\frac{V_{1}^{n}}{\hbar \omega}\right) e^{-i(E+m \hbar \omega) t / \hbar},
\end{aligned}
$$

where

$$
k^{n}(E)=\frac{\sqrt{2 m^{*}\left(E-V_{0}^{n}\right)}}{\hbar},
$$

and $J_{m}$ is a Bessel function of the first kind. The coefficients $A(E)$ and $B(E)$ are determined by boundary conditions. These coefficients should be zero at $E \neq E_{0}+p \hbar \omega$, where $E_{0}$ is the energy of the incident electron and $p=0, \pm 1, \pm 2, \ldots$ because of the boundary condition at $x \rightarrow-\infty$ and the form of Eq. (2.3) itself. This implies that the energy of the electron can be changed only by way of absorbing or emitting $p$ energy quanta of $\hbar \omega$. Therefore, the solution $\psi^{n}(x, t)$ is written as

$$
\begin{aligned}
\psi^{n}(x, t)= & \sum_{p=-\infty}^{\infty}\left[A(p) e^{i k^{n}(p) x}+B(p) e^{-i k^{n}(p) x}\right] \\
& \times \sum_{m=-\infty}^{\infty} J_{m}\left(\frac{V_{1}^{n}}{\hbar \omega}\right) \exp \left[-i\left(E_{0}+p \hbar \omega+m \hbar \omega\right) t / \hbar\right],
\end{aligned}
$$

where $A(p), B(p)$, and $k^{n}(p)$ are the abbreviated notations of $A\left(E_{0}+p \hbar \omega\right), B\left(E_{0}+p \hbar \omega\right)$, and $k^{n}\left(E_{0}+p \hbar \omega\right)$, respectively. The boundary conditions to determine the coefficients $A(p)$ and $B(p)$ are

$$
\begin{gathered}
\psi^{n}\left(x^{n}, t\right)=\psi^{n+1}\left(x^{n}, t\right), \\
\partial_{x} \psi^{n}\left(x^{n}, t\right)=\partial_{x} \psi^{n+1}\left(x^{n}, t\right) .
\end{gathered}
$$

These conditions should be valid for any time $t$. Substituting Eq. (2.5) into Eq. (2.6), we obtain

$$
\begin{gathered}
\sum_{p=-\infty}^{\infty} \phi_{+}^{n}\left(p, x^{n}\right) J_{r-p}\left(\frac{V_{1}^{n}}{\hbar \omega}\right) \\
=\sum_{p=-\infty}^{\infty} \phi_{+}^{n+1}\left(p, x^{n}\right) J_{r-p}\left(\frac{V_{1}^{n+1}}{\hbar \omega}\right), \\
\sum_{p=-\infty}^{\infty} k^{n}(p) \phi_{-}^{n}\left(p, x^{n}\right) J_{r-p}\left(\frac{V_{1}^{n}}{\hbar \omega}\right) \\
=\sum_{p=-\infty}^{\infty} k^{n+1}(p) \phi_{-}^{n+1}\left(p, x^{n}\right) J_{r-p}\left(\frac{V_{1}^{n+1}}{\hbar \omega}\right),
\end{gathered}
$$

for any integer $r$. Here

$$
\phi_{ \pm}^{n}(p, x)=A^{n}(p) e^{i k^{n}(p) x} \pm B^{n}(p) e^{-i k^{n}(p) x} .
$$

Using the addition theorem for the Bessel functions,

$$
\sum_{m^{\prime}=-\infty}^{\infty} J_{m+m^{\prime}}(x) J_{m^{\prime}}(y)=J_{m}(x-y),
$$

Eq. (2.7) reads

$$
\phi_{+}^{n+1}\left(p, x^{n}\right)=\sum_{q=-\infty}^{\infty} \phi_{+}^{n}\left(q, x^{n}\right) J_{p-q}\left(\frac{V_{1}^{n}-V_{1}^{n+1}}{\hbar \omega}\right),
$$

$$
\phi_{-}^{n+1}\left(p, x^{n}\right)=\sum_{q=-\infty}^{\infty} \frac{k^{n}(q)}{k^{n+1}(p)} \phi_{-}^{n}\left(q, x^{n}\right) J_{p-q}\left(\frac{V_{1}^{n}-V_{1}^{n+1}}{\hbar \omega}\right) .
$$

From Eqs. (2.8) and (2.10), the coefficients $A^{n+1}$ and $B^{n+1}$ are expressed by $A^{n}$ and $B^{n}$ as

$$
\begin{aligned}
A^{n+1}(p)= & \sum_{q=-\infty}^{\infty}\left[g^{n}(p, q ; 1,-1) A^{n}(p+q)\right. \\
& \left.+g^{n}(p, q ;-1,-1) B^{n}(p+q)\right], \\
B^{n+1}(p)= & \sum_{q=-\infty}^{\infty}\left[g^{n}(p, q ;-1,1) A^{n}(p+q)\right. \\
& \left.+g^{n}(p, q ; 1,1) B^{n}(p+q)\right],
\end{aligned}
$$

where

$$
\begin{aligned}
g^{n}(p, q ; \alpha, \beta)= & \frac{1}{2}\left[1+\alpha \frac{k^{n}(p+q)}{k^{n+1}(p)}\right] \exp \left\{i \beta \left[k^{n+1}(p)\right.\right. \\
& \left.\left.-\alpha k^{n}(p+q)\right] x^{n}\right\} J_{-q}\left(\frac{V_{1}^{n}-V_{1}^{n+1}}{\hbar \omega}\right) .
\end{aligned}
$$

Using Eq. (2.11), in principle, one can obtain $A^{N}(p)$ and $B^{N}(p)$ from $A^{1}(p)$ and $B^{1}(p)$. In actual numerical calculations, the summations in Eq. (2.11) should be truncated at a finite order. Coefficients $A^{n}(p)$ and $B^{n}(p)$ are, however, small compared with $A^{n}\left(p^{\prime}\right)$ and $B^{n}\left(p^{\prime}\right)$, respectively, if $|p|<\left|p^{\prime}\right|$, because $\left|V_{1}^{n}-V_{1}^{n+1}\right| \ll \hbar \omega$ for sufficiently small 
segments and $J_{n}(x)$ for $x \ll 1$ is a monotonically decreasing function of $|n|$. This corresponds to the fact that the probability to absorb (emit) $p$ energy quanta of the oscillating external field is larger than that for $p^{\prime}$ quanta if $p<p^{\prime}$. In order to calculate the coefficients $A^{n}(p)$ and $B^{n}(p)$ within the $M$ th-order approximation in which $M$ quanta excitations are taken into account, we restrict the ranges of $p$ and $q$ in Eq. (2.11) as

$$
\begin{gathered}
|p| \leqslant M, \\
|q| \leqslant M, \\
|p+q| \leqslant M .
\end{gathered}
$$

The similar expression to Eq. (2.11) has been presented with the aim of constructing a transfer matrix for a onedimensional system $[9,23,24]$. However, the phase factor in Eq. (2.12) and the way to approximate were not properly evaluated in previous works.

It is convenient to define a vector $Y_{\mu}^{n}(\mu=1,2, \ldots, 4 M$ +2 ) defined by

$$
Y_{\mu}^{n}= \begin{cases}A^{n}\left(M-\frac{\mu-1}{2}\right) & \text { if }(\mu \bmod 2)=1 \\ B^{n}\left(M-\frac{\mu-2}{2}\right) & \text { if }(\mu \bmod 2)=0 .\end{cases}
$$

Using the vector $\boldsymbol{Y}^{n}$, Eq. (2.11) can be written in a matrix form:

$$
\boldsymbol{Y}^{n+1}=\widetilde{\Lambda}^{n} \boldsymbol{Y}^{n}
$$

where $\widetilde{\Lambda}^{n}$ is a $(4 M+2) \times(4 M+2)$ matrix. Hereafter the tilde on a symbol implies that the symbol is a matrix. The matrix $\widetilde{\Lambda}^{n}$ is expressed by a $(2 M+1) \times(2 M+1)$ block matrix whose elements are given by $2 \times 2$ submatrices. The block matrix element $\tilde{\Lambda}_{l l^{\prime}}^{n}\left(l, l^{\prime}=1,2, \ldots, 2 M+1\right)$ of the matrix $\widetilde{\Lambda}^{n}$ is then

$$
\widetilde{\Lambda}_{l l^{\prime}}^{n}=\widetilde{\xi}^{n}\left(l-l^{\prime}, M-l+1\right),
$$

where

$$
\widetilde{\xi}^{n}(p, q)=\eta(p) \eta(q) \widetilde{\theta}^{n}(p, q)
$$

Here $\eta(p)$ is a step function defined by

$$
\eta(p)= \begin{cases}1 & \text { if }|p| \leqslant M \\ 0 & \text { if }|p|>M\end{cases}
$$

and $\widetilde{\theta}^{n}(p, q)$ is a $2 \times 2$ matrix:

$$
\widetilde{\theta}^{n}(p, q)=\left(\begin{array}{cc}
g^{n}(p, q ; 1,-1) & g^{n}(p, q ;-1,-1) \\
g^{n}(p, q ;-1,1) & g^{n}(p, q ; 1,1)
\end{array}\right) .
$$

The matrix element $\Lambda_{\mu \mu^{\prime}}^{n}\left(\mu, \mu^{\prime}=1,2, \ldots, 4 M+2\right)$ is, then, given by

$$
\Lambda_{\mu \mu^{\prime}}^{n}=\eta(p) \eta(q) g^{n}(p, q ; \alpha, \beta)
$$

where $p=[(\mu-1) / 2]-\left[\left(\mu^{\prime}-1\right) / 2\right], \quad q=[M-(\mu-1) / 2]$ $+1, \alpha=1-2\left\{\left(\mu+\mu^{\prime}\right) \bmod 2\right\}$, and $\beta=1-2(\mu \bmod 2)$. The symbol $[x]$ denotes the largest integer less than or equal to $x$. For instance, in the case of $M=2$, the $10 \times 10$ matrix $\widetilde{\Lambda}^{n}$ reads

$$
\widetilde{\Lambda}^{n}=\left(\begin{array}{ccccc}
\widetilde{\theta}^{n}(0,2) & \widetilde{\theta}^{n}(-1,2) & \widetilde{\theta}^{n}(-2,2) & 0 & 0 \\
\widetilde{\theta}^{n}(1,1) & \widetilde{\theta}^{n}(0,1) & \widetilde{\theta}^{n}(-1,1) & \widetilde{\theta}^{n}(-2,1) & 0 \\
\widetilde{\theta}^{n}(2,0) & \widetilde{\theta}^{n}(1,0) & \widetilde{\theta}^{n}(0,0) & \widetilde{\theta}^{n}(-1,0) & \widetilde{\theta}^{n}(-2,0) \\
0 & \widetilde{\theta}^{n}(2,-1) & \widetilde{\theta}^{n}(1,-1) & \widetilde{\theta}^{n}(0,-1) & \widetilde{\theta}^{n}(-1,-1) \\
0 & 0 & \widetilde{\theta}^{n}(2,-2) & \widetilde{\theta}^{n}(1,-2) & \widetilde{\theta}^{n}(0,-2)
\end{array}\right)
$$

We construct a transfer matrix $\widetilde{\mathcal{T}}$ that relates the left-hand side coefficients vector $\boldsymbol{Y}^{1}$ to the right-hand side one $\boldsymbol{Y}^{N}$ as $\boldsymbol{Y}^{N}=\widetilde{\mathcal{T}} \boldsymbol{Y}^{1}$ by

$$
\widetilde{\mathcal{T}}=\prod_{n=1}^{N} \widetilde{\Lambda}^{n}
$$

Since $\boldsymbol{Y}^{1}$ has its nonzero components only for the incident wave and reflecting waves,

$$
Y_{\mu}^{1}= \begin{cases}B^{1}\left(M-\frac{\mu-2}{2}\right) & \text { if }(\mu \bmod 2)=0 \\ 1 & \text { if } \mu=2 M+1 \\ 0 & \text { otherwise. }\end{cases}
$$

Moreover, $\boldsymbol{Y}^{N}$ involves only amplitudes of transmitting waves;

$$
Y_{\mu}^{N}= \begin{cases}A^{N}\left(M-\frac{\mu-1}{2}\right) & \text { if }(\mu \bmod 2)=1 \\ 0 & \text { otherwise. }\end{cases}
$$


Substituting these conditions into the relation $\boldsymbol{Y}^{N}=\widetilde{\mathcal{T}} \boldsymbol{Y}^{1}$, one can easily solve $A^{N}$ and $B^{1}$ as

$$
\begin{aligned}
A^{N}(p)= & \mathcal{T}_{2 M-2 p+1,2 M+1} \\
& \quad-\sum_{l=1}^{2 M+1} \sum_{l^{\prime}=1}^{2 M+1} \mathcal{T}_{2 M-2 p+1,2 l} \Gamma_{l l^{\prime}} \mathcal{T}_{2 l^{\prime}, 2 M+1}, \\
& B^{1}(p)=-\sum_{l=1}^{2 M+1} \Gamma_{M-p+1, l} \mathcal{I}_{2 l, 2 M+1}
\end{aligned}
$$

where the $(2 M+1) \times(2 M+1)$ matrix $\widetilde{\Gamma}$ is the inverse matrix of $\mathcal{T}_{2 l, 2 l^{\prime}}\left(l, l^{\prime}=1,2, \ldots, 2 M+1\right)$, i.e.,

$$
\sum_{l^{\prime \prime}=1}^{2 M+1} \Gamma_{l l^{\prime \prime}} \mathcal{T}_{2 l^{\prime \prime}, 2 l^{\prime}}=\delta_{l l^{\prime}}
$$

Finally the transmission and reflection probabilities through the $p$ th sideband are given by

$$
T(p)=\frac{k^{R}(p)}{k^{L}(0)}\left|A^{N}(p)\right|^{2}
$$

and

$$
R(p)=\frac{k^{L}(p)}{k^{L}(0)}\left|B^{1}(p)\right|^{2},
$$

respectively, where

$$
\begin{gathered}
k^{L}(p)=\frac{\sqrt{2 m^{*}\left(E_{0}+p \hbar \omega\right)}}{\hbar}, \\
k^{R}(p)=\frac{\sqrt{2 m^{*}\left(E_{0}+V_{0}(\infty)+p \hbar \omega\right)}}{\hbar} .
\end{gathered}
$$

The probability conservation law, $\sum_{p=-M}^{M}[T(p)+R(p)]$ $=1$, is valid only in the limiting case of $M \rightarrow \infty$, and the quantity $\Sigma_{p=-M}^{M}[T(p)+R(p)]$ becomes less than unity for a finite value of $M$. The deviation $\Delta \equiv 1-\sum_{p=-M}^{M}[T(p)$ $+R(p)]$ is, however, quite small, if $M$ is sufficiently large. In fact, the order of $\Delta$ is $\left|N J_{M+1}(z)\right|^{2}$, where $N$ is the number of segments into which the system is divided, $z$ $=V_{1}^{\max } / N \hbar \omega$, and $V_{1}^{\max }$ is the maximum value of $\left|V_{1}(x)\right|$. The quantity $\left|N J_{M+1}(z)\right|^{2}$ is a decreasing function of $M$ and $N$. This means that $\Delta$ becomes small even for small $M$ if one chooses sufficiently large $N$. For instance, in the case that $V_{1}^{\max }=\hbar \omega, N=100$, and $M=3$, the deviation $\Delta$ is smaller than $10^{-18}$.

\section{TWO-DIMENSIONAL SYSTEMS}

In this section, we extend our transfer matrix method to two-dimensional systems. The formulation is essentially the same as the one-dimensional one except for additional channels of transport due to the transverse degree of freedom. The two-dimensional version of the transfer matrix method should be able to extract the electron transport through a specific sideband and a channel. The method for two dimen- sions can be easily extended to a three-dimensional one, because multichannel transport in three-dimensional systems has no intrinsic difference from a two-dimensional one.

Here we concentrate on the case where the static potential $V_{0}$ is an arbitrary function of $x$ and $y$ while the amplitude of the ac potential $V_{1}$ depends only on $x$. The restriction on $V_{1}$ does not greatly reduce the applicability of the present method. The Schrödinger equation of an electron in such a system is

$$
\begin{aligned}
i \hbar \frac{\partial}{\partial t} \psi(x, y, t)= & -\frac{\hbar^{2}}{2 m^{*}}\left(\frac{\partial^{2}}{\partial x^{2}}+\frac{\partial^{2}}{\partial y^{2}}\right)+V_{0}(x, y) \\
& \left.+V_{1}(x) \cos \omega t\right] \psi(x, y, t) .
\end{aligned}
$$

An example of physical systems described by Eq. (3.1) is a two-dimensional electron in the presence of a linearly polarized electromagnetic field, which propagates in the direction perpendicular to the two-dimensional electron surface. Choosing the $x$ and $z$ directions as the polarization and propagating directions of the electromagnetic wave, respectively, the ac potential $V_{1}$ by the electromagnetic wave does not depend on $y$. Since the oscillating magnetic field, induced by the electromagnetic wave, in the $y$ direction cannot affect the orbital motion of the electron in the $x-y$ plane, the electron in this system is described by Eq. (3.1).

Hereafter we consider the electron transport in the $x$ direction. In order to formulate the transfer matrix method, the system is divided, in the $x$ direction, into $N$ thin strips with a width of $\Delta x$. As in the one-dimensional case, we assume that $\Delta x$ is so small that $V_{0}$ and $V_{1}$ can be regarded as constants with respect to $x$ within each strip. In the $n$th strip, the wave function is governed by

$$
\begin{aligned}
i \hbar \frac{\partial}{\partial t} \psi^{n}(x, y, t)= & -\frac{\hbar^{2}}{2 m^{*}}\left(\frac{\partial^{2}}{\partial x^{2}}+\frac{\partial^{2}}{\partial y^{2}}\right)+V_{0}^{n}(y) \\
& \left.+V_{1}^{n} \cos \omega t\right] \psi^{n}(x, y, t),
\end{aligned}
$$

where $V_{0}^{n}$ and $V_{1}^{n}$ are defined in the same way as in the one-dimensional case. The special solution of this equation is

$$
\psi_{ \pm}^{n}(x, y, t)=e^{ \pm i k^{n} x} \varphi^{n}(y) \sum_{m=-\infty}^{\infty} J_{m}\left(\frac{V_{1}^{n}}{\hbar \omega}\right) e^{-i(E+m \hbar \omega) t / \hbar},
$$

where $E$ is a constant, $\varphi^{n}(y)$ satisfies

$$
\left[-\frac{\hbar^{2}}{2 m^{*}} \frac{\partial^{2}}{\partial y^{2}}+V_{0}^{n}(y)\right] \varphi^{n}(y)=\varepsilon^{n} \varphi^{n}(y),
$$

and $k^{n}=\sqrt{2 m^{*}\left(E-\varepsilon^{n}\right)} / \hbar$. Equation (3.4) can be solved numerically by discretizing the space in the $y$ direction into $K$ grid points $y_{\kappa}(\kappa=1,2, \ldots, K)$. One obtains $K$ solutions of 
Eq. (3.4), which are denoted by $\varphi_{\nu}^{n}$ and $\varepsilon_{\nu}^{n}(\nu=1,2, \ldots, K)$ for each $n$. The wave functions $\varphi_{\nu}^{n}\left(y_{\kappa}\right)$ are chosen to be orthonormalized as

$$
\sum_{\kappa=1}^{K} \varphi_{\nu}^{n}\left(y_{\kappa}\right) \varphi_{\nu^{\prime}}^{n}\left(y_{\kappa}\right)=\delta_{\nu \nu^{\prime}} \quad\left(\nu, \nu^{\prime}=1,2, \ldots, K\right) .
$$

Using the set of these wave functions, the general solution of Eq. (3.2) in the discretized space is given by

$$
\begin{aligned}
\psi^{n}\left(x, y_{\kappa}, t\right)= & \sum_{\nu=1}^{K} \sum_{p=-\infty}^{\infty}\left[A_{\nu}^{n}(p) e^{i k_{\nu}^{n}(p) x}+B_{\nu}^{n}(p) e^{-i k_{\nu}^{n}(p) x}\right] \\
& \times \varphi_{\nu}^{n}\left(y_{\kappa}\right) \sum_{m=-\infty}^{\infty} J_{m}\left(\frac{V_{1}^{n}}{\hbar \omega}\right) e^{-i\left(E_{0}+p \hbar \omega+m \hbar \omega\right) t / \hbar},
\end{aligned}
$$

where

$$
k_{\nu}^{n}(p)=\frac{\sqrt{2 m^{*}\left(E_{0}+p \hbar \omega-\varepsilon_{\nu}^{n}\right)}}{\hbar},
$$

and $A_{\nu}^{n}$ and $B_{\nu}^{n}$ are amplitudes of right- and left-going waves, respectively.

From the matching conditions, $\psi^{n}\left(x^{n}, y_{\kappa}, t\right)$ $=\psi^{n+1}\left(x^{n}, y_{\kappa}, t\right)$ and $\partial_{x} \psi^{n}\left(x^{n}, y_{\kappa}, t\right)=\partial_{x} \psi^{n+1}\left(x^{n}, y_{\kappa}, t\right)$, the coefficients $A_{\nu}^{n+1}$ and $B_{\nu}^{n+1}$ can be expressed by $A_{\nu}^{n}$ and $B_{\nu}^{n}$ as

$$
\begin{aligned}
A_{\nu}^{n+1}(p)= & \sum_{\nu^{\prime}=1}^{K} \sum_{q=-\infty}^{\infty}\left[g_{\nu \nu^{\prime}}^{n}(p, q ; 1,-1) A_{\nu^{\prime}}^{n}(p+q)\right. \\
& \left.+g_{\nu \nu^{\prime}}^{n}(p, q ;-1,-1) B_{\nu^{\prime}}^{n}(p+q)\right], \\
B_{\nu}^{n+1}(p)= & \sum_{\nu^{\prime}=1}^{K} \sum_{q=-\infty}^{\infty}\left[g_{\nu \nu^{\prime}}^{n}(p, q ;-1,1) A_{\nu^{\prime}}^{n}(p+q)\right. \\
& \left.+g_{\nu \nu^{\prime}}^{n}(p, q ; 1,1) B_{\nu^{\prime}}^{n}(p+q)\right],
\end{aligned}
$$

where

$$
\begin{aligned}
g_{\nu \nu^{\prime}}^{n}(p, q ; \alpha, \beta)= & \frac{1}{2}\left[1+\alpha \frac{k_{\nu^{\prime}}^{n}(p+q)}{k_{\nu}^{n+1}(p)}\right] C_{\nu \nu^{\prime}}^{n} \exp \left\{i \beta \left[k_{\nu}^{n+1}(p)\right.\right. \\
& \left.\left.-\alpha k_{\nu^{\prime}}^{n}(p+q)\right] x^{n}\right\} J_{-q}\left(\frac{V_{1}^{n}-V_{1}^{n+1}}{\hbar \omega}\right),
\end{aligned}
$$

and

$$
C_{\nu \nu^{\prime}}^{n}=\sum_{\kappa=1}^{K} \varphi_{\nu}^{n+1}\left(y_{\kappa}\right) \varphi_{\nu^{\prime}}^{n}\left(y_{\kappa}\right)
$$

Here we use the orthonormality condition Eq. (3.5) and the addition theorem for the Bessel functions Eq. (2.9). The quantity $C_{\nu \nu^{\prime}}^{n}$ represents a channel mixing between the $n$th and $(n+1)$ th strips. In actual calculations within the $M$ th-order approximation, the ranges of $p$ and $q$ in Eq. (3.8) are restricted by Eq. (2.13). As in the case of onedimensional problems, we introduce a coefficient vector $\boldsymbol{Y}^{n}$ to define the transfer matrix. For two-dimensional problems, $\boldsymbol{Y}^{n}$ has $(4 M+2) K$ elements due to $K$ channels of the transport. Each element of $\boldsymbol{Y}^{n}$ is given by

$$
Y_{\mu}^{n}=\left\{\begin{array}{ll}
A_{\nu}^{n}(M-\zeta) & \text { if } \lambda=1 \\
B_{\nu}^{n}(M-\zeta) & \text { if } \lambda=0
\end{array} \quad \mu=1,2, \ldots,(4 M+2) K,\right.
$$

where

$$
\begin{gathered}
\zeta=\left[\frac{\mu-1}{2 K}\right], \\
\lambda=\left(\left[\frac{\mu+K-1}{K}\right] \bmod 2\right), \\
\nu=\{(\mu-1) \bmod K\}+1 .
\end{gathered}
$$

Equation (3.8) can be expressed by using this vector as

$$
\boldsymbol{Y}^{n+1}=\tilde{\Lambda}^{n} \boldsymbol{Y}^{n} .
$$

$\widetilde{\Lambda}^{n}$ is a $(4 M+2) K \times(4 M+2) K$ matrix. Dividing this matrix into $2 K \times 2 K$ submatrices, the $\left(l, l^{\prime}\right)$ element of the block matrix is given by

$$
\tilde{\Lambda}_{l l^{\prime}}^{n}=\widetilde{\xi}^{n}\left(l-l^{\prime}, M-l+1\right), \quad l, l^{\prime}=1,2, \ldots, 2 M+1,
$$

where

$$
\widetilde{\xi}^{n}(p, q)=\eta(p) \eta(q) \widetilde{\theta}^{n}(p, q),
$$

and the $2 K \times 2 K$ matrix $\widetilde{\theta}^{n}(p, q)$ is

$$
\widetilde{\theta}^{n}(p, q)=\left(\begin{array}{cc}
\widetilde{G}^{n}(p, q ; 1,-1) & \widetilde{G}^{n}(p, q ;-1,-1) \\
\widetilde{G}^{n}(p, q ;-1,1) & \widetilde{G}^{n}(p, q ; 1,1)
\end{array}\right) .
$$

The function $\eta(p)$ was defined by Eq. (2.18). Here the $K \times K$ matrix $\widetilde{G}^{n}(p, q ; \alpha, \beta)$ has its $\left(\nu, \nu^{\prime}\right)$ element as $g_{\nu \nu^{\prime}}^{n}(p, q ; \alpha, \beta)$. Therefore the matrix element $\Lambda_{\mu \mu^{\prime}}^{n}\left(\mu, \mu^{\prime}\right.$ $=1,2, \ldots,(4 M+2) K)$ is expressed as

$$
\Lambda_{\mu \mu^{\prime}}^{n}=\eta(p) \eta(q) g_{\nu \nu^{\prime}}^{n}(p, q ; \alpha, \beta),
$$

where $p=[(\mu-1) / 2 K]-\left[\left(\mu^{\prime}-1\right) / 2 K\right], q=M-[(\mu-1)$ $/ 2 K]+1, \quad \nu=\{(\mu-1) \bmod K\}+1, \quad \nu^{\prime}=\left\{\left(\mu^{\prime}-1\right) \bmod K\right\}$ $+1, \quad \alpha=1-2\left\{\left([(\mu-1) / K]+\left[\left(\mu^{\prime}-1\right) / K\right]\right) \bmod 2\right\}, \quad$ and $\beta=1-2([(\mu-1) / 2] \bmod 2)$.

The coefficient vector $\boldsymbol{Y}^{N}$ of the $N$ th strip is calculated from $\boldsymbol{Y}^{1}$ of the first strip by the matrix $\widetilde{\mathcal{T}}=\prod_{n=1}^{N} \widetilde{\Lambda}^{n}$. The elements of $\boldsymbol{Y}^{1}$ and $\boldsymbol{Y}^{N}$ are given by

$$
Y_{\mu}^{1}= \begin{cases}B_{\nu}^{1}(M-\zeta) & \text { if } \lambda=0 \\ D_{\nu} & \text { if } \mu=2 M k+\nu \\ 0 & \text { otherwise, }\end{cases}
$$


and

$$
Y_{\mu}^{N}= \begin{cases}A_{\nu}^{N}(M-\zeta) & \text { if } \lambda=1 \\ 0 & \text { otherwise }\end{cases}
$$

where $\zeta, \lambda$, and $\nu$ are the same as those defined by Eq. (3.12) and $D_{\nu}$ is the $\nu$ th channel amplitude of the incident wave. By solving the equation $\boldsymbol{Y}^{N}=\widetilde{\mathcal{T}} \boldsymbol{Y}^{1}$ under the conditions (3.18) and (3.19), the coefficients $A_{\nu}^{N}$ and $B_{\nu}^{1}$ are expressed as

$$
\begin{aligned}
& \boldsymbol{A}^{N}(p)= \widetilde{W}_{2 M-2 p+1,2 M+1} \boldsymbol{D} \\
&-\sum_{l, l^{\prime}=1}^{2 M+1} \widetilde{W}_{2 M-2 p+1,2 l} \widetilde{\Gamma}_{l l^{\prime}} \widetilde{W}_{2 l^{\prime}, 2 M+1} \boldsymbol{D}, \\
& \boldsymbol{B}^{1}(p)=-\sum_{l=1}^{2 M+1} \widetilde{\Gamma}_{M-p+1, l} \widetilde{W}_{2 l, 2 M+1} \boldsymbol{D},
\end{aligned}
$$

where $\boldsymbol{A}^{N}(p), \boldsymbol{B}^{1}(p)$, and $\boldsymbol{D}$ are vectors whose elements are $A_{\nu}^{N}(p), \quad B_{\nu}^{1}(p), \quad$ and $D_{\nu} \quad(\nu=1,2, \ldots, K)$, respectively. $\widetilde{W}_{\gamma \gamma^{\prime}}$ 's $\left(\gamma, \gamma^{\prime}=1,2, \ldots, 4 M+2\right)$ are $K \times K$ matrices into which the $(4 M+2) K \times(4 M+2) K$ matrix $\widetilde{\mathcal{T}}$ is divided. $\widetilde{\Gamma_{l l^{\prime}}}$ is a $K \times K$ matrix defined by

$$
\sum_{l^{\prime \prime}=1}^{2 M+1} \widetilde{\Gamma}_{l l^{\prime \prime}} \widetilde{W}_{2 l^{\prime \prime}, 2 l^{\prime}}=\widetilde{\Delta}_{l l^{\prime}},
$$

where $\widetilde{\Delta_{l l^{\prime}}}$ is a $K \times K$ matrix whose $\left(\nu, \nu^{\prime}\right)$ element is $\delta_{l l^{\prime}} \delta_{\nu \nu^{\prime}}$. The transmission probability $T_{\nu}(p)$ through the $p$ th sideband and the $\nu$ th channel is, for example, given by $T_{\nu}(p)=k_{\nu}^{L}(p)\left|A_{\nu}^{N}(p)\right|^{2} / \sum_{\nu=1}^{K} k_{\nu}^{R}(0)\left|D_{\nu}\right|^{2}$, where $\quad k_{\nu}^{L}(p)=\sqrt{2 m^{*}\left(E_{0}+p \hbar \omega-\varepsilon_{\nu}^{L}\right)} / \hbar$ and $k_{\nu}^{R}(p)$ $=\sqrt{2 m^{*}\left(E_{0}+p \hbar \omega-\varepsilon_{\nu}^{R}\right)} / \hbar . \varepsilon_{\nu}^{L}$ and $\varepsilon_{\nu}^{R}$ are the eigenenergies in Eq. (3.4) with $V_{0}(y)=V_{0}(\infty)$ and $V_{0}(y)=V_{0}(-\infty)=0$, respectively.

\section{APPLICATION TO DYNAMICAL STARK EFFECT IN A DOUBLE BARRIER PAT DEVICE}

In this section, we apply the transfer matrix method to electron transport through a double barrier structure in a microwave irradiation. This process is a typical example of the photon-assisted tunneling [7-16]. PAT is an electron transport phenomenon exchanging the energy of an external electromagnetic field. This phenomenon has been found in superconductor-insulator-superconductor tunneling junctions $[7,8]$, semiconductor superlattices $[11,12]$, or twodimensional quantum well devices [13-16]. Here we examine our transfer matrix method by applying it to PAT phenomena in a double barrier structure in which electron transport can be regarded to be one dimensional. Such a system can be realized by the two-dimensional electron gas (2DEG) in a dual-gate field-effect transistor fabricated by GaAs-AlGaAs [14]. Gates with strip shapes are configured parallel to the transverse direction (the direction perpendicular to the electron transport). The irradiated field is applied perpendicular to the 2DEG surface, and the polarization of the ac field is in the longitudinal direction. If the transverse

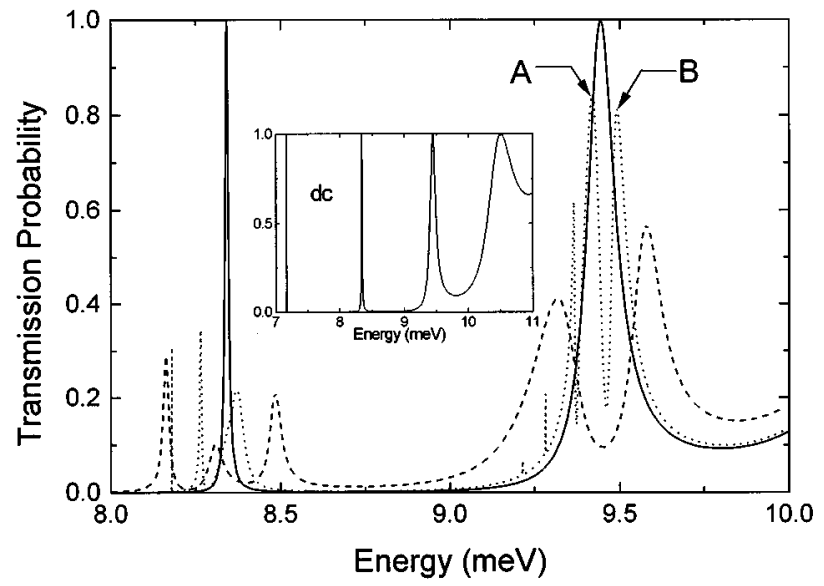

FIG. 1. Transmission probabilities for double-barrier systems $\left(V_{0}=10 \mathrm{meV}, x_{0}=150 \mathrm{~nm}\right.$, and $\left.\xi=50 \mathrm{~nm}\right)$ with and without an ac field as a function of the energy of the incident electron. Solid lines in this figure and its inset indicate the transmission probability for the static system. Dotted line shows the result for the harmonically driven system ( $\hbar \omega=1.10 \mathrm{meV}$ and $\alpha=1)$. Dashed line indicates the ac transmission probability calculated by setting $M=1$.

dimension of the system is sufficiently large, the electron transport is described by the one-dimensional Schrödinger equation (2.1).

We will show that the system exhibits the dynamical Stark effect by applying a strong ac field. The dynamical Stark effect is a level splitting phenomenon observed when the frequency of the ac field is made resonant with the energy separation between two levels of the system, and was studied for atomic systems more than forty years ago [25]. Our numerical calculations presented in this section show that the dynamical Stark effect can occur even in a semiconductor PAT device.

The potential $V_{0}(x)$ and $V_{1}(x)$ in Eq. (2.1) are chosen as

$$
V_{0}(x)=V_{0}\left[e^{-\left(x+x_{0}\right)^{2} / \xi^{2}}+e^{-\left(x-x_{0}\right)^{2} / \xi^{2}}\right]
$$

and

$$
V_{1}(x)=-e \mathcal{E} x
$$

respectively, where $e$ and $\mathcal{E}$ in Eq. (4.2) are the electron charge and the strength of the electric field. Here parameters in Eqs. (2.1) and (4.1) are set to be $V_{0}=10 \mathrm{meV}, x_{0}=150$ $\mathrm{nm}, \xi=50 \mathrm{~nm}$, and $m^{*}=0.067 m_{e}$. These values are not far from those in experimental situations [14]. The total length of this one-dimensional system is $1 \mu \mathrm{m}$. In order to carry out the transfer matrix calculations, the system is divided into 200 segments, so the length of each segment is $5 \mathrm{~nm}$. This length is sufficiently small in comparison with $\xi$ and a typical electron wavelength $\lambda_{e}\left(\lambda_{e} \sim 50 \mathrm{~nm}\right.$, when $\left.E=10 \mathrm{meV}\right)$. At first, we calculated a dc transmission probability by using our transfer matrix method with $V_{1}(x)=0$. The result shown in the inset of Fig. 1 exhibits that the system has several resonant tunneling levels. Since the static potential barriers are symmetric around $x=0$, transmission probabilities at these resonant levels are exactly equal to unity.

In order to obtain transmission probabilities in ac fields, the frequency of the ac field is chosen to be equal to the 


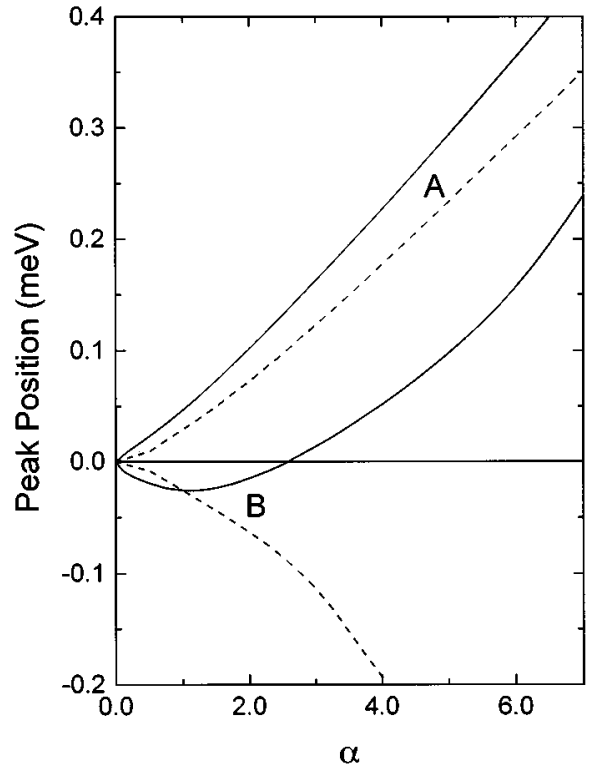

FIG. 2. Peak positions of split levels vs the ac field strength. Solid lines $A$ and $B$ are the energy shifts (from $E_{2}$ ) of peaks labeled by $A$ and $B$ on the dotted line in Fig. 1, respectively. Dashed lines show peak positions of the dashed line in Fig. 1.

energy difference between two adjacent resonant levels $E_{1}$ and $E_{2}$, where $E_{1}=8.34 \mathrm{meV}$ and $E_{2}=9.44 \mathrm{meV}$, so $\hbar \omega$ $=1.10 \mathrm{meV}$. It is convenient to introduce a quantity $\alpha$ defined by $\alpha=2 e \mathcal{E} x_{0} / \hbar \omega$, which represents the strength of the electromagnetic field. The actual magnitude of the electric field is, for example, $\mathcal{E}=37 \mathrm{~V} / \mathrm{cm}$ for $\alpha=1$. The dotted line in Fig. 1 shows the ac transmission probability for $\alpha=1$. In this calculation, the order of the approximation, $M$ in Eq. (2.13), is 6 . The violation of the probability conservation (unitarity) is of the order of $10^{-11}$. The dotted line indicates that the resonant tunneling energy $E_{2}$ is split into several levels.

This level splitting can be attributed to the dynamical Stark effect. The theory of the dynamical Stark effect for a two level system without any dissipation was formulated by Autler and Townes [25]. In their theory, a system were supposed to be moleculelike, so the energy levels $\left(E_{1}\right.$ and $\left.E_{2}\right)$ are treated as perfectly sharp. In this case, the level is split into doublets $\left(\varepsilon_{+}\right.$and $\left.\varepsilon_{-}\right)$symmetrically around the original level. The width of the energy splitting $\Delta \varepsilon=\left|\varepsilon_{+}-\varepsilon_{-}\right|$is given by

$$
\Delta \varepsilon=2 w\left[1-\frac{w^{2}}{8\left(E_{1}-E_{2}\right)^{2}}\right],
$$

where $w$ is the matrix element of the potential $e \mathcal{E} x$ between two states at $E_{1}$ and $E_{2}$. The above expression is precise within third order of $w$. The Rabi splitting [26] for the case of a magnetic dipole in a rotating magnetic field is equivalent to this phenomenon $[27,28]$. The symmetric splitting has also been found in a system described by a tunneling Hamiltonian with only two resonant levels [29]. On the other hand, our system possesses many resonant levels and energy differences between adjacent levels are close each other. (The energy of the lower adjacent level of $E_{1}$ is 7.17 and $10.50 \mathrm{meV}$

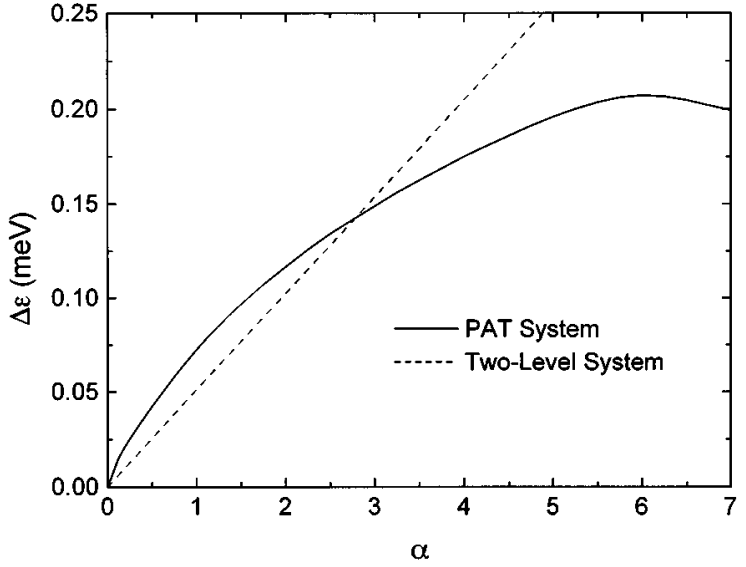

FIG. 3. Energy separation $\Delta \varepsilon$ of split levels as a function of the ac field strength. Solid line indicates $\Delta \varepsilon$ between resonant peaks labeled by $A$ and $B$ in Fig. 1. Dashed line is calculated in terms of Eq. (4.3) with $d=7 \mathrm{~nm}$.

for the upper adjacent level of $E_{2}$.) In this case, as shown in Fig. 1, the original level is split into more than two, and the splitting is not symmetric. The dashed line in Fig. 1 shows the transmission probability calculated by setting $M=1$. This result extracts mainly the effect of excitations between only two levels $E_{1}$ and $E_{2}$. It should be noted that the result, of course, violates the unitarity. The fact that in the dashed line the original level is split into doublets makes it clear that the multiple splitting is attributed to the existence of many resonant levels separated by almost equal distance. Figure 2 indicates the $\alpha$ dependence of split level energies. The solid lines are results for peaks labeled by $A$ and $B$ on the dotted line in Fig. 1. The dashed lines in Fig. 2 show peak positions of the dashed line in Fig. 1. The symmetric character of the dashed lines suggests that the asymmetric splitting (the solid lines in Fig. 2) is due to an asymmetric profile of the dc transmission probability around $E=E_{1}$.

The energy separation $\Delta \varepsilon$ between split levels labeled by $A$ and $B$ in Fig. 1 is plotted by solid line in Fig. 3 as a function of the field strength $\alpha$. For small $\alpha(\alpha \lesssim 3)$, the width of the split levels is roughly proportional to the square root of the field strength. However, $\Delta \varepsilon$ decreases for $\alpha>6$. According to a simple Tien-Gordon picture [7], the amplitude of a one-photon absorption sideband state is proportional to $J_{1}\left(\alpha^{*}\right)$, where $\alpha^{*}$ is an effective strength of the external ac field. Therefore, the decreasing of $\Delta \varepsilon$ can be explained by the oscillating character of the Bessel function. ( $\alpha^{*} \sim 0.3 \alpha$ from the maximum point of the $\Delta \varepsilon$-curve.) The dashed line in Fig. 3 is calculated in terms of Eq. (4.3), which is valid only for a system with two perfectly sharp levels. The quantity $w$ in Eq. (4.3) is written as $w=e \mathcal{E} d$, where $d$ is an off-diagonal element of $x$. The value of $d$ for the dashed line was chosen as $7 \mathrm{~nm}$. The numerical result for our PAT system obviously differs from Eq. (4.3). This discrepancy is a consequence of many resonant levels in our system.

Results of our numerical calculations suggest that the level splitting caused by the dynamical Stark effect occurs even in an irradiated semiconductor PAT device, as in an atomic system. The computing time is about $1.2 \mathrm{sec}$ to calculate a transmission probability at a specific incident energy 
of electron by using our transfer matrix method and a computer facility of FACOM VPP500, while the CPU time is more than $1 \mathrm{~h}$ for the same calculation by solving numerically the time-dependent Schrödinger equation. This fact clearly shows the efficiency of our method.

\section{CONCLUSIONS}

A transfer matrix method has been developed for investigating quantum transport in dynamical mesoscopic systems with harmonically oscillating potentials. Since the energy conservation law is violated in dynamical mesoscopic systems, a conventional transfer matrix technique cannot be applied. Using the present numerical method, one can obtain transmission and reflection probabilities of systems with arbitrary dc and ac potentials. (In a two-dimensional case, the spatial profile of the ac potential should depend only on the longitudinal direction.) This method saves much computing time compared with solving numerically a time-dependent Schrödinger equation. The CPU time required for the transfer matrix calculation is proportional to $N(4 M+2)^{3}$ and $N K^{3}(4 M+2)^{3}$ for one- and two-dimensional systems, respectively. Here $N$ and $K$ are numbers of divisions of the system in the $x$ and $y$ directions, respectively, and $M$ is the order of the approximation introduced by Eq. (2.13). The proportionality coefficient depends on neither a potential profile nor an energy of the incident electron, but on the performance of a computer hardware. The value of this coefficient, for example, for FACOM VPP500 is about 3.4 $\times 10^{-7}$ sec. Consequently, one can calculate a transmission probability by using the transfer matrix method more than a thousand times faster than by the numerical integration of the Schrödinger equation. The memory space for the calculation is proportional to $(4 M+2)^{2}$ and $K^{2}(4 M+2)^{2}$ for one- and two-dimensional systems, respectively. The precision of a calculated result depends on $N, K$, and $M$. In particular, the unitarity condition does not hold for insufficient $M$, but $K$ is irrelevant to this condition. The required value of $M$ is determined by the strength of the ac potential and the number of segments $N$.

We applied our numerical method to a PAT in a dual-gate field-effect transistor. Results suggest that the resonant level splitting due to the dynamical Stark effect occurs in an irradiated semiconductor PAT device. The level splitting has the following characteristic features: (i) The resonant energy is split into several levels, not into doublets. (ii) The level splitting is asymmetric. (iii) The width of the energy splitting does not obey Eq. (4.3). These features arise from the existence of many resonant levels separated by almost equal distance and the asymmetry of the level distribution around a specific level, and are significant for characterizing the PAT device as an artificial atom. The fact that these results can be obtained within a very short CPU time proves the efficiency of our numerical method.

The transfer matrix methods for one- and twodimensional systems have been described in this paper. The extension to a three-dimensional system is easy. We hope that the present method will be utilized to find or study new transport phenomena in dynamical mesoscopic systems.

\section{ACKNOWLEDGMENTS}

We would like to thank T. Nakayama, T. Tezuka, J. Ohe, and $\mathrm{Q}$. Hu for stimulating discussions. Numerical calculations were performed partially on the FACOM VPP500 of Supercomputer Center, Institute for Solid State Physics, University of Tokyo. This work was supported in part by a Grant-in-Aid for Scientific Research from the Ministry of the Education, Science, and Culture of Japan (No. 09233201).
[1] Nano-structures and Mesoscopic Systems, edited by W. P. Kirk and M. A. Reed (Academic Press, San Diego, 1992).

[2] Proceedings of the 11th International Conference on Electronic Properties of Two-Dimensional Systems, edited by L. Eaves and P. C. Main [Surf. Sci. 361-362 (1996)].

[3] M. Holthaus, G. H. Ristow, and D. W. Hone, Phys. Rev. Lett. 75, 3914 (1995).

[4] M. El Ghafar, P. Törmä, V. Savichv, E. Mayr, A. Zeiler, and W. P. Schleich, Phys. Rev. Lett. 78, 4181 (1997).

[5] B. J. Keay, S. Zeuner, S. J. Allen, Jr., K. D. Maranowski, A. C. Gossard, U. Bhattacharya, and M. J. W. Rodwell, Phys. Rev. Lett. 75, 4102 (1995).

[6] T. Nakanishi, T. Ohtsuki, and T. Kawarabayashi, J. Phys. Soc. Jpn. 66, 949 (1997).

[7] P. K. Tien and J. R. Gordon, Phys. Rev. 129, 647 (1963).

[8] Q. Hu, C. A. Mears, P. L. Richards, and F. L. Lloyd, Phys. Rev. B 42, 10250 (1990).

[9] H. C. Liu, Phys. Rev. B 43, 12538 (1991).

[10] H. C. Liu, M. Buchanan, and Z. R. Wasilewski, Phys. Rev. B 44, 1411 (1991).

[11] P. F. Hopkins, A. C. Gossard, L. T. Florez, and J. P. Harbison,
Phys. Rev. Lett. 70, 3792 (1993).

[12] B. J. Keay, S. J. Allen, Jr., J. Galan, J. P. Kaminki, K. L. Campman, A. C. Gossard, U. Bhattacharya, and M. J. W. Rodwell, Phys. Rev. Lett. 75, 4098 (1995).

[13] L. P. Kouwenhoven, S. Jauhar, J. Orenstein, P. L. McEuen, Y. Nagamune, J. Motohisa, and H. Sakaki, Phys. Rev. Lett. 73, 3443 (1994).

[14] S. Verghese, R. A. Wyss, Th. Schäpers, Q. Hu, A. Förster, and M. J. Rooks, Phys. Rev. B 52, 14834 (1995).

[15] Q. Hu, S. Verghese, R. A. Wyss, Th. Schäpers, J. del Alamo, S. Feng, K. Yakubo, M. J. Rooks, M. R. Melloch, and A. Förster, Semicond. Sci. Technol. 11, 1888 (1996).

[16] T. H. Oosterkamp, L. P. Kouwenhoven, A. E. A. Koolen, N. C. van der Vaart, and C. J. P. M. Harmans, Phys. Rev. Lett. 78, 1536 (1997).

[17] W. Cai, T. F. Zheng, P. Hu, B. Yudanin, and M. Lax, Phys. Rev. Lett. 63, 418 (1989).

[18] H. Chen, Y. Shi, J. Yu, Jia-Lin Zhu, and Y. Kawazoe, Phys. Rev. B 55, 9935 (1997).

[19] R. Landauer, IBM J. Res. Dev. 1, 223 (1957).

[20] R. Landauer, Philos. Mag. 21, 863 (1970). 
[21] K. Yakubo, S. Feng, and Q. Hu, Phys. Rev. B 54, 7987 (1996).

[22] J. Crank and P. Nicolson, Proc. Cambridge Philos. Soc. 32, 50 (1947).

[23] D. D. Coon and H. C. Liu, J. Appl. Phys. 58, 2230 (1985).

[24] H. C. Liu, Superlattices Microstruct. 12, 17 (1992).

[25] S. H. Autler and C. H. Townes, Phys. Rev. 100, 703 (1955).
[26] I. I. Rabi, Phys. Rev. 51, 652 (1932).

[27] C. A. Stafford and Ned S. Wingreen, Phys. Rev. Lett. 76, 1916 (1996).

[28] A. Liu, Phys. Rev. B 55, 7101 (1997).

[29] P. Johansson and G. Wendin, Phys. Rev. B 46, 1451 (1992). 